\title{
Heuristic Enhancement of Magneto-Optical Images for NDE
}

\author{
Matteo Cacciola, Giuseppe Megali, Diego Pellicanò, Salvatore Calcagno, \\ Mario Versaci, and Francesco Carlo Morabito
}

\author{
DIMET Department, Faculty of Engineering, University "Mediterranea" of Reggio Calabria, Via Graziella Feo di Vito, \\ 89100 Reggio Calabria, Italy
}

Correspondence should be addressed to Matteo Cacciola, matteo.cacciola@unirc.it

Received 31 December 2009; Revised 15 May 2010; Accepted 26 August 2010

Academic Editor: João Marcos A. Rebello

Copyright () 2010 Matteo Cacciola et al. This is an open access article distributed under the Creative Commons Attribution License, which permits unrestricted use, distribution, and reproduction in any medium, provided the original work is properly cited.

\begin{abstract}
The quality of measurements in nondestructive testing and evaluation plays a key role in assessing the reliability of different inspection techniques. Each different technique, like the magneto-optic imaging here treated, is affected by some special types of noise which are related to the specific device used for their acquisition. Therefore, the design of even more accurate image processing is often required by relevant applications, for instance, in implementing integrated solutions for flaw detection and characterization. The aim of this paper is to propose a preprocessing procedure based on independent component analysis (ICA) to ease the detection of rivets and/or flaws in the specimens under test. A comparison of the proposed approach with some other advanced image processing methodologies used for denoising magneto-optic images (MOIs) is carried out, in order to show advantages and weakness of ICA in improving the accuracy and performance of the rivets/flaw detection.
\end{abstract}

\section{Introduction}

For both improving manufacturing quality and guaranteeing safety, devices, components, and structures are usually inspected to detect the presence of defects or faults which may threaten their integrity. Nondestructive testing and evaluation (NDT\&E) are industrial methodologies which couple the ability of detecting defects and characterizing them usually by means of noninvasive procedures [1]. In experimental NDT\&E, the measurements can give details on the structural properties of the sample [2], like cracks, flaws and phase transformations that may develop in discontinuous deformations. Typically, the main challenge is to detect and characterize flaws starting from experimental measurements through the solution of suitably formulated inverse problems. Because of the limitations of the measurement recording and the presence of noise, the problem to be solved through inversion is commonly ill-posed as well as ill-conditioned in its numerical counterpart. The critical decision for a pattern recognition system is the selection of appropriate features to be extracted from the image for classification. These features should be unique, informative, and invariant to translation and rotation of the image. In aircraft structures the rivets are of different sizes and so it is important that the features should also be size invariant. Therefore, emphasizing informative pattern in the data set by filtering the noise is strictly required in order to obtain suitable input data for the inverse problem solution. Within this framework, the main concern is the implementation of automated solutions able to support scientists in characterizing defects by using nondestructive analysis. This difficulty can be overcome by using the recent advances in computing power and signal processing techniques. A number of different approaches on interpreting the eddy current sensor response using advanced signal processing techniques have been proposed $[3,4]$. In this paper, we propose some advanced image processing approaches able to solve a particular problem for enhancing MOI. It is also critical to keep the information unchanged or unperturbed by the applied noise filtering techniques. Otherwise, the inverse problem solution will produce ambiguous results [5].

MOI [6] is a relatively new technology, based in part on the Faraday rotation effect and used in nondestructive inspection. MOI is being used extensively in inspection of 
aircraft fuselage skin for detecting subsurface corrosion and cracks at rivet sites. In aircraft inspection, data (images) from a large number of rivets need to be analysed. Currently it is manually performed by scanning the MO imager over the inspection surface. The main advantage of MOI (see $[7,8]$ about MOI designed by physical research instruments (PRI)) can inspect large areas at high speed compared with classical eddy current methods. Other advantages are its fast and easy inspection capabilities in comparison with other conventional nondestructive inspection instruments. An automated real-time method for evaluating the MO images for structural defects can reduce human error and increase accuracy and speed of the inspections. Such a method requires the elimination of background (image) noise due to presence of magnetic domain walls in the MO sensor and enhancing the image for subsequent interpretation. This paper presents an image processing and automated classification algorithm that classifies the MO images for both surface and subsurface cracks in aircraft skins under various excitation frequencies. Within this framework, progresses have been made in developing the MOI for detection of subsurface cracks and corrosion by improvements in instrument design. The key to the instrument's capability in detecting the relatively weak magnetic fields associated with subsurface defects is the sensitivity of the MO sensor. The goal of this work is to introduce an enhanced MOI-based approach for imaging magnetic fields of NDT\&E. Within this framework, tests have been carried out and the presented methods have been evaluated by experimental results. The paper is organized as follows. Section 2 briefly describes the basic principles of MOI, with a particular overview of domain noise in MO images. Section 3 introduces and deepens the denoising of MO images by using two image processing techniques: the former is based on ICA [9-11], the latter is a self-implemented adaptive homomorphic filter (AHF). Both were compared with the motion-based filtering (MBF) $[12,13]$, a well-known technique able to reduce static noise in images. Finally, in Section 4, the performances of the proposed approaches are discussed.

\section{Principles of MOI Technique}

The MOI technology depends on exciting the aircraft skin by eddy current induction and measuring the normal magnetic field component. The time-varying magnetic field of the AC current passing through the planar induction foil induces a sheet of eddy current from its uniform flow and hence generates magnetic field perpendicular to the surface of the aircraft skin. The normal magnetic field component is measured using the Faraday rotation effect where a linearly polarized light transmitted through as MO garnet sensor is rotated by an amount proportional to the magnetic field $\mathbf{H}$. Devices use an induction coil for inducing eddy currents in a conducting sample and an MO sensor for detecting the magnetic flux density associated with the induced eddy currents. In a defect-free specimen, the induced current is uniform and the associated magnetic field lies in the plane of the sensor. Anomalies in the specimen, such as fasteners or surface and subsurface cracks, result in generating a magnetic flux density normal to the sensor plane. If the linearly polarized light is incident to the sensor, the polarization plane of light will be rotated by an angle $\theta$ that is proportional to the sensor thickness, given approximately by [7]

$$
\theta \approx \theta_{f} \frac{(\bar{K} \cdot \bar{M})}{\|\bar{K}|\|\mid \bar{M}\|},
$$

where $\bar{K}$ is the wave vector of the incident light, $\bar{M}$ is the local magnetization of the epitaxial layer (sensor) at the point where the incident light passes through the layer, and $\theta_{f}$ is the incident angle of light. The sign of the scalar product $\bar{K} \cdot \bar{M}$ determines the direction of the rotation. For more information about MOI, the interested reader could refer to $[12,13]$ and references therein. A MO sensor generates a realtime analog image of the magnetic field associated with the induced eddy current interacting with structural anomalies. The resulting images are formed by distortion within the magnetic domain of the MO sensor, as a reaction to the external field. Images are relatively insensitive to the field intensity and can be reproduced by a charge-coupled device (CCD) camera on a monitor. MO imaging can be used to detect defects in both ferromagnetic and non-ferromagnetic materials. The sensitivity of the resulting images depends on the levels of the induced eddy current, operating frequency, and sensor parameters. The used technique, jointly with the key features of magnetic materials, generates domain noise, such as mazes, bubble lattices, and strips [14]. A schematic of the MOI instrument is shown in Figure 1. A copper foil is used to produce uniform sheet currents at low frequency $(1.5-100[\mathrm{kHz}])$, which induces eddy currents in the conducting test specimen. Under normal conditions, the associated magnetic flux is tangential to specimen surface. Cracks in the specimen generate a normal component of the magnetic flux density. When a linearly polarized light is incident normally on the sensor, the plane of polarization of the light is rotated by the angle $\theta$. This angle is decided by the normal component of the magnetic flux density applied on the sensor. When the reflected light is viewed through the analyser, local occurrence of normal magnetic flux is seen as a "dark" or "light" area in the magneto-optic image depending on the direction of magnetization.

Usually, MOI inspection is conducted by a human operator by scanning the surface of sample with the device. The MOI data is interpreted by the operator in real time or recorded as a video sequence for later interpretation. This leads to variability in flaw detection according to the experience of inspector. Here we propose some methods exploiting sequences of MOI for enhancing the quality of MO images and the corresponding inspection capabilities. Figure 2 shows three consecutive frames in an MOI scanning video. The dark disks represent rivets of $0.3 \mathrm{~cm}$ in diameter and the dark bands show a substrate seam into the airplane structure. The dark areas on the left are due to the leakage of uncompensated magnetic field on the edge of the inspecting area. Figure 2 shows that the background components are stationary from frame to frame while objects in the sample are moving. The scanning direction goes to left, thus 


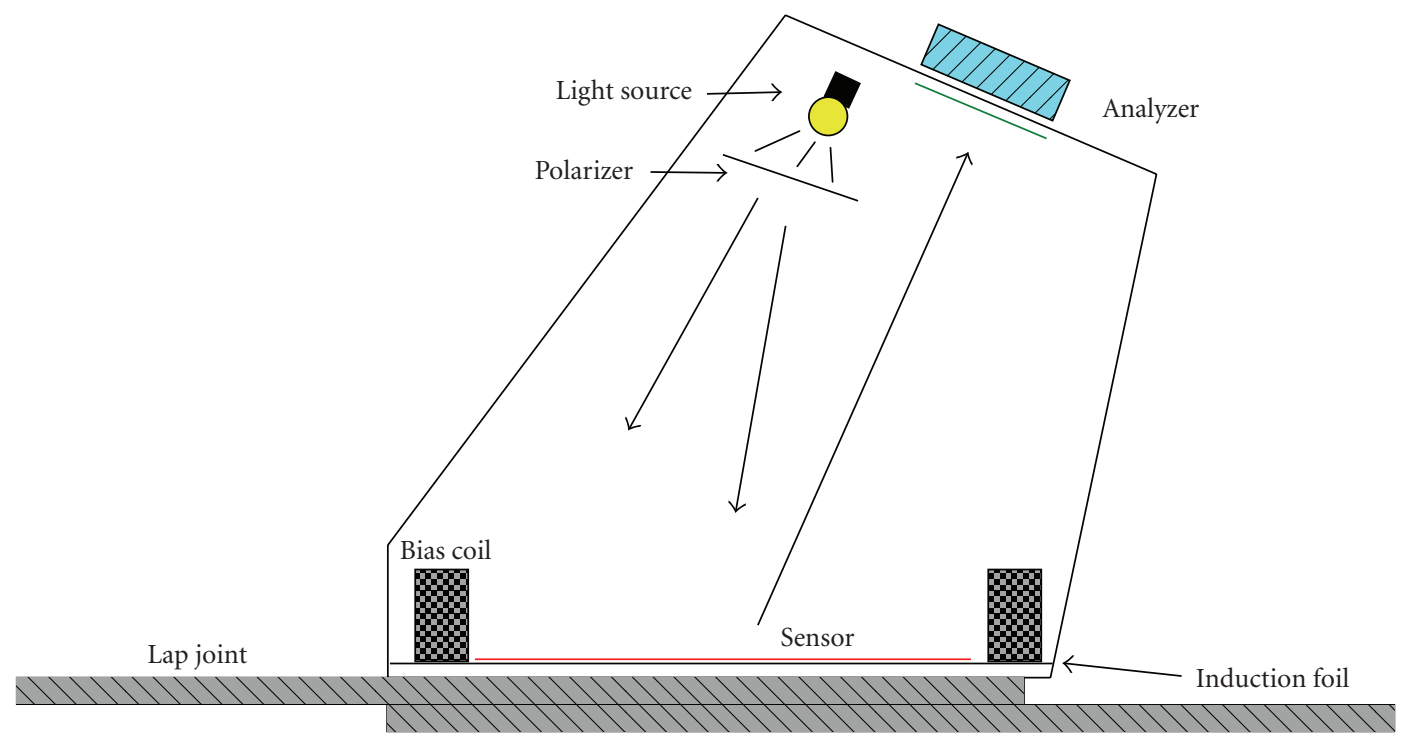

FIGURE 1: Schematic of the magneto optic imaging system.

the rivets move to the right during the scanning. Generally speaking, MO images data can be divided into two components: dynamic foreground and stationary background. Usually, the foreground image is considered as the signal and the background as noise to be suppressed.

2.1. Domain Noise in MOI. Magnetic materials, as previously mentioned, exhibit many types of domain structures, such as mazes, bubble lattices, and strips, which are affected by various factors: the magnetic anisotropy and the magnetization of the material, its shape, the presence of defects, the external magnetic field, temperature, surface treatment, and the previous history of the sample [14]. In the MOI, the domain structures introduce complex artifacts hardly removable by means of naïve techniques, like using simple image thresholding and filtering. Furthermore, passivating techniques (i.e., anodizing and alodizing) respond in a different way to the external excitation. Another source of noise and degradation in MO data is due to magnetic domain boundaries in the garnet sensor. The domain boundaries appear as a small, filament-like structure in MO images (Figure 3) and can severely mask the presence of cracks and corrosion.

The presence of this textured background in MO images due to domain structures makes detection of cracks and corrosion difficult. However, due to the magnetic domain wall structures of the sensor, the MO images could be corrupted by a characteristic noise, which could decrease the MOI inspection capabilities. The domain walls generally produce serpentine pattern noise, which can be reduced by improving the sensor or by use of image processing methods [13]. The noise hinders detection of small cracks and corrosion located in the second and third layers, limiting the capability of inspection. This leads to the need for an image processing algorithm for reducing this background noise. In MO images, noise associated with the domain structures in the sensor is overall stationary, since it is related to the stationary background of images. In fact, since the sample is scanned using the MOI device, the domain noise resident in the sensor moves with the sensor and hence is stationary with respect to the sensor. In contrast, rivets and cracks in the sample appear to move from frame to frame. Therefore, algorithms presented in this paper particularly aim to separate the moving parts from the stationary parts in the sequence of images without generating distortions. Bubbles, mazes, and other background static noises, due to the tape itself, can be thought as non-Gaussian distributions, convolved with the useful signal. In this way, the challenge of denoising MOI can be approached as distinguishing nonGaussian distributions, comprising the useful signals. In this way, the challenge of denoising MOI can be approached as a problem of blind source separation (BSS) exploiting higherorder statistical features. The raw images, resulting from the MO image acquisition system, have been processed by a BSS technique such as ICA and by means of an adaptive filtering technique such as AHF. Performances of these techniques have been compared with MBF, a well-known technique which is reputed to yield good detection performance.

\section{Removing Background Noise in MOI}

Generally speaking, any measurement device is disturbed by parasitic phenomena. This includes the electronic noise and also external events that affect the measured phenomenon, depending on what is measured and on the sensitivity of device. It is often possible to reduce the noise by controlling the environment; otherwise, when the characteristics of the noise are known or are different from the signal ones, it is possible to filter it or to process the signal. Particularly, in our case we exploited a Matlab code, according to Young et al. [15], in order to calculate image SNR performances, before and after the filtering step. 


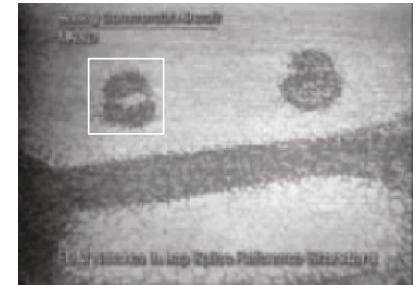

Frame 125

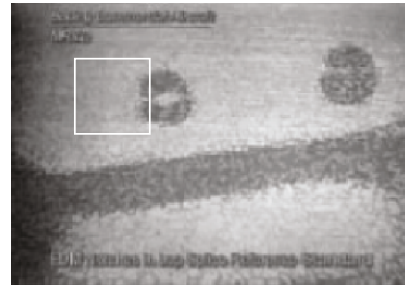

Frame 126

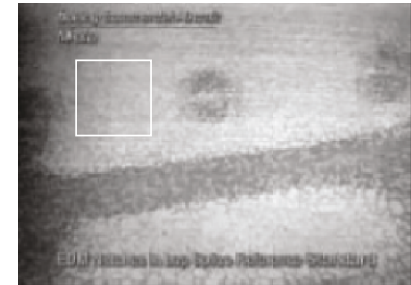

Frame 127

Scanning direction of MOI instrument

Figure 2: Three consecutive MOI images; dark disks represent rivets, which are moving to the right, while the sensor is moving to the left. Dark bands show a substrate seam into the airplane structure.

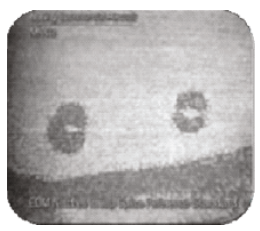

Frame 33

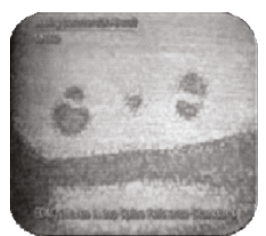

Frame 36

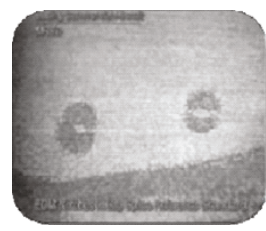

Frame 34

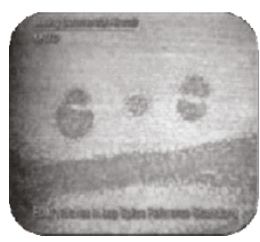

Frame 37

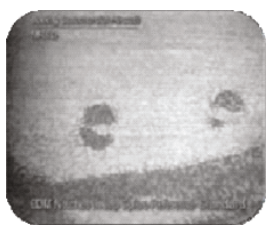

Frame 35
Figure 3: Frames grabbed from one of the available experimental sets.

Within this framework, we will process a set of various MO images that have been collected by a commercial MOI 301 apparatus at the NDE Lab, Michigan State University, USA [12].

3.1. Presentation of the Collected Data. Data exploited for our experimentation have been collected on a set of fatigue crack lap splice samples consisting of 36 panels with 720 rivet sites containing first-layer fatigue cracks of various sizes. The induction frequency used was $50[\mathrm{kHz}]$. MOI data was directly recorded on a tape during the acquisition, and subsequently has been acquired into a personal computer system and saved as an AVI file. The movie has the characteristics resumed in Table 1.

We selected six different sets, composed by five different but consecutive frames per set, in order to evaluate and compare the different exploited techniques: ICA, a selfimplemented AHF, and MBF. Our aim is to propose signalprocessing methods for eliminating the noise due to domain boundaries and enhancing the signals due to objects of interest. Figure 4 shows a particular MO image with two rivets. The left rivet is unflawed whilst the rivet on the right hand shows a radial crack. We can see that the normal rivet image is roughly circular in shape while the abnormal rivet is noncircular.

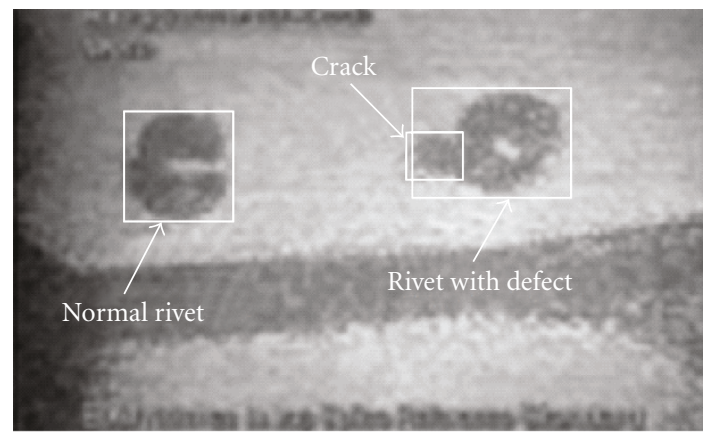

FIGURE 4: Sample MO image. On the left, a normal rivet with a circular shape; on the right, a rivet with crack in its site.

Figure 5 depicts the exported sets. The reason of composing each set by 5 different frames will be described within the next subsection. As it is possible to denote, the sets cover the whole time length of the movie, in order to give as much generality as possible to the proposed approach. Moreover, let us remark how, on each set, the images composing the set itself show the same number of objects, that is, rivets, and the same objects, except for the 5th set: frames 127, 128, and 129 show, in fact, different number of objects and/or just different objects. The 5 th set has been voluntarily added to the experimental set, in order to evaluate the ICA's performance in similar applicative contexts.

3.2. ICA for Enhancing MOI. The problem of source separation has been deeply analysed in electrical engineering; many algorithms exist, depending also on the nature of the mixed signals. The problem faced by the BSS is more difficult because it is not possible to design appropriate preprocessors in order to optimally separate mixed signals without any knowledge about them. Even in NDT\&E it is possible to meet problems involving mixed signals and BSS. For instance, let us consider the problem of noisy measurements in MOI: in this framework, noise is an additive effect with respect to the useful information. In many cases of practical relevance, often for the presence of nonlinear phenomena, or when a noise source is not strictly gaussian (e.g., the lift-off effect in eddy current testing), it is very difficult to separate the informative signal from the uninformative one. In this case, 


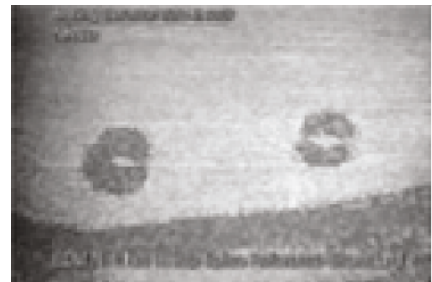

Frame 33

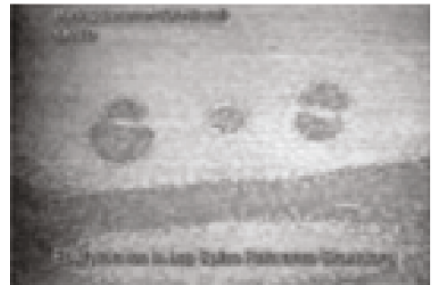

Frame 36

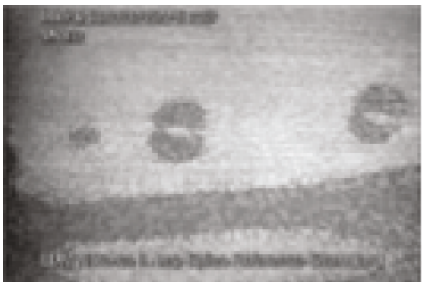

Frame 50

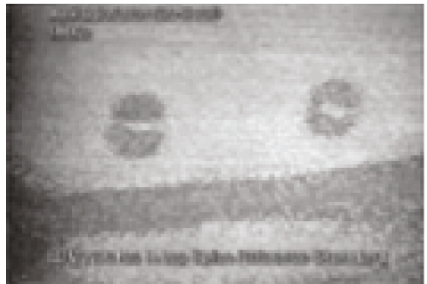

Frame 53

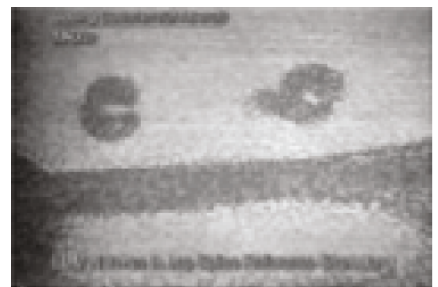

Frame 75

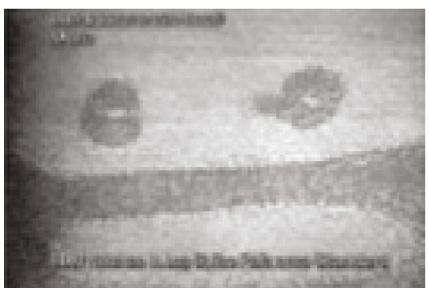

Frame 78

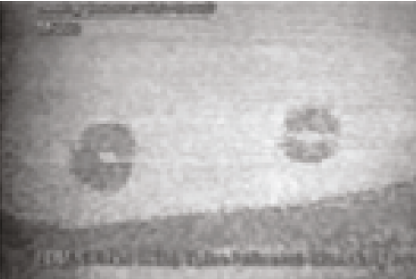

Frame 34

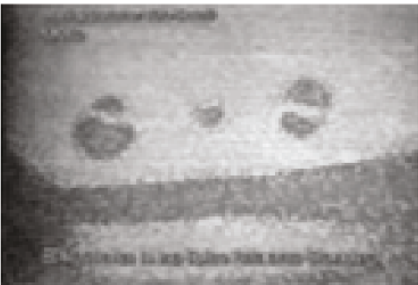

Frame 37

(a) 1 st set

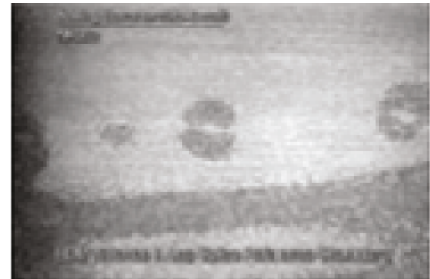

Frame 51

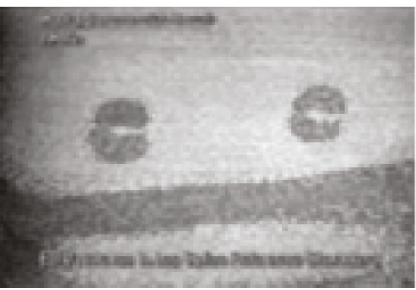

Frame 54

(b) 2nd set

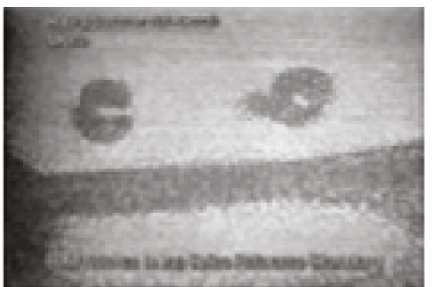

Frame 76

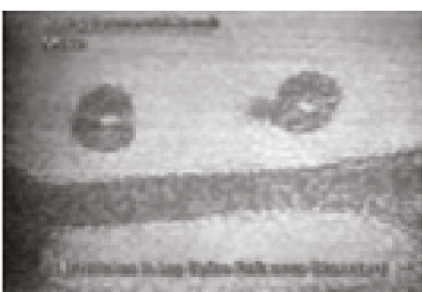

Frame 79

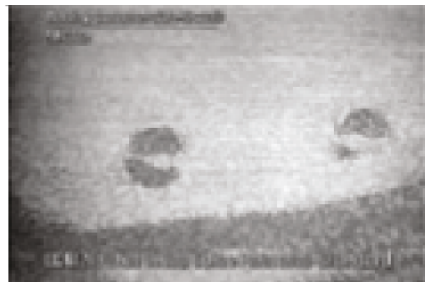

Frame 35

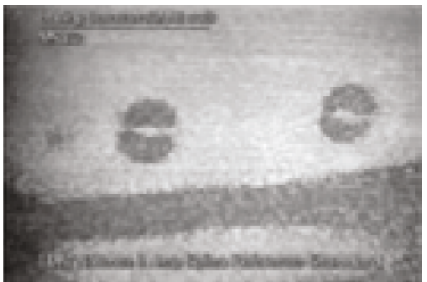

Frame 52

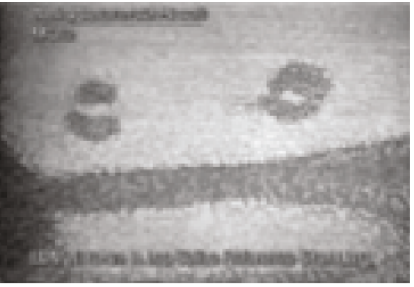

Frame 77

(c) 3rd set

Figure 5: Continued. 


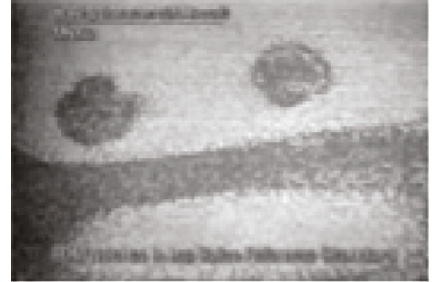

Frame 115

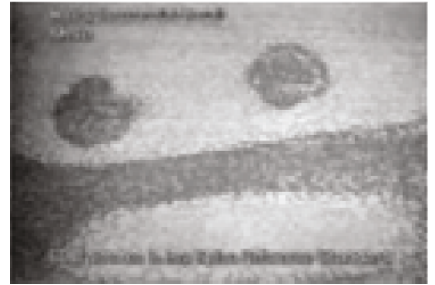

Frame 118

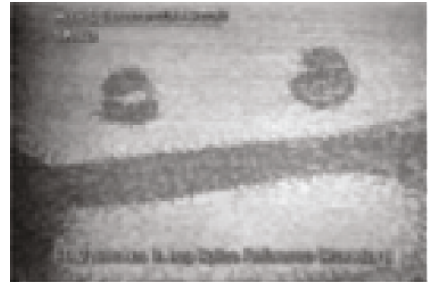

Frame 125

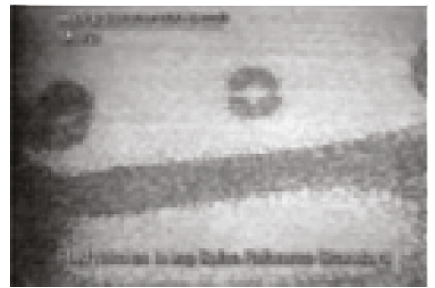

Frame 128

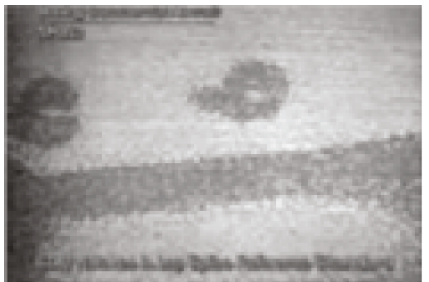

Frame 166

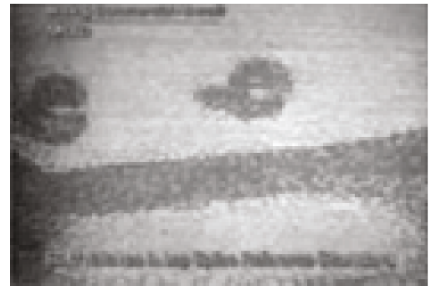

Frame 169

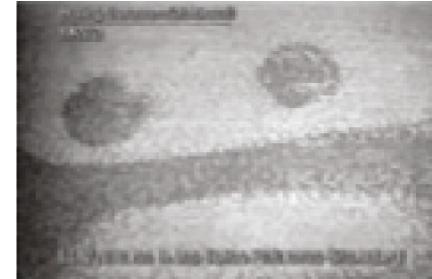

Frame 116

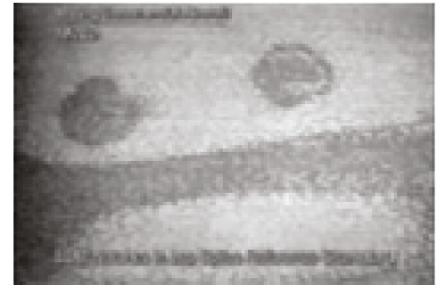

Frame 119

(d) 4 th set

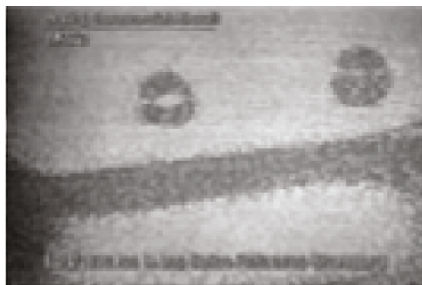

Frame 126

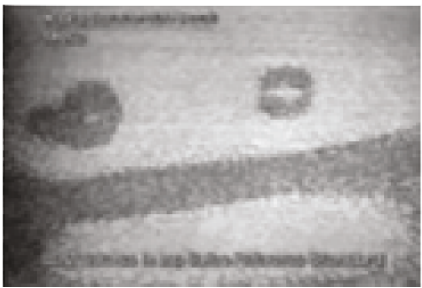

Frame 129

(e) 5 th set

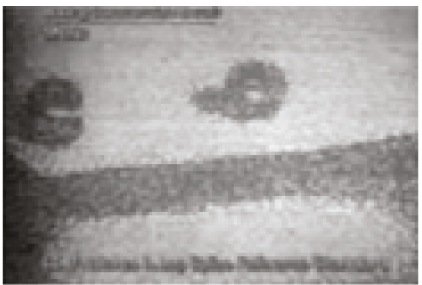

Frame 167

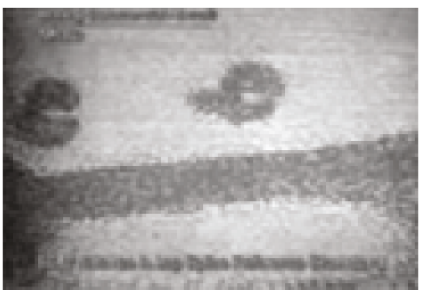

Frame 170

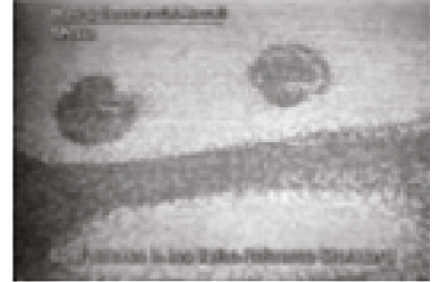

Frame 117

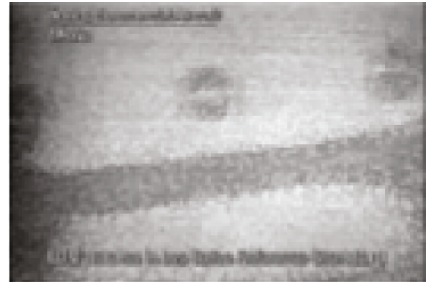

Frame 127

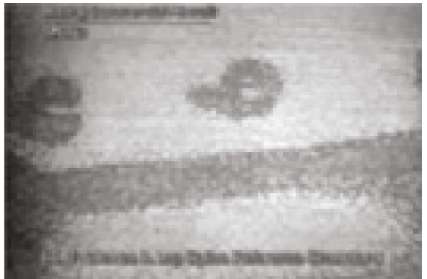

Frame 168

(f) 6th set

FIGURE 5: The set of magneto-optic images exploited in this experimentation. 
TABLE 1: Characteristics of the AVI movie file exploited in this experimentation.

\begin{tabular}{lccc}
\hline Time length & Rate & Resolution & Codec \\
\hline $17[\mathrm{~s}]$ & $367[\mathrm{Kbps}], 10.0[\mathrm{fps}]$ & $320 * 240(4: 3)$ & MS-MPEG standard (MPEG4) \\
\hline
\end{tabular}

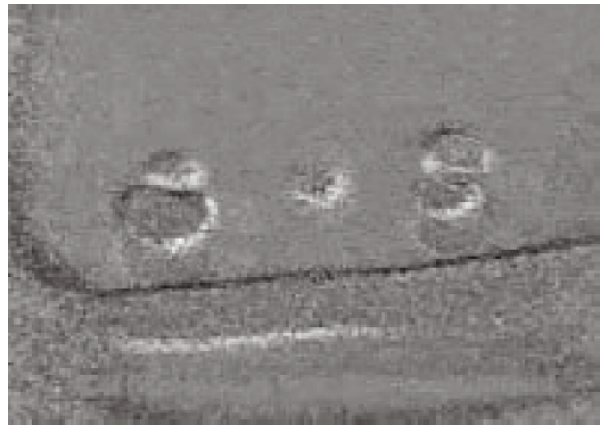

(a) 1st set: useful component

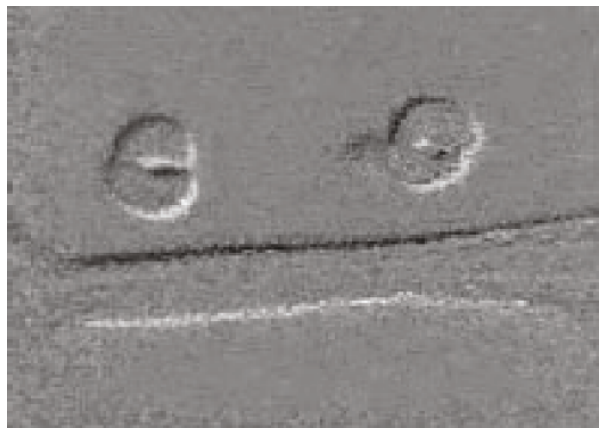

(c) 3rd set: useful component

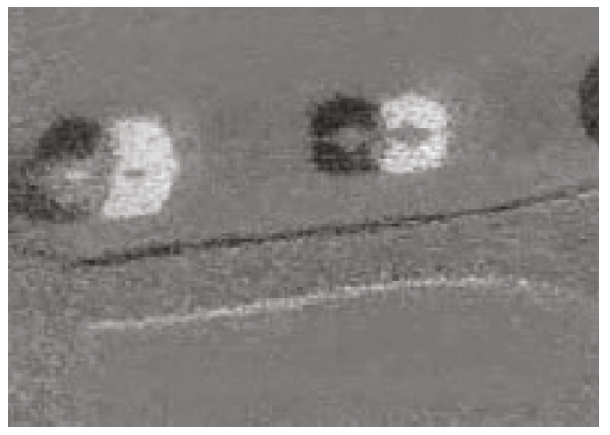

(e) 5th set: useful component

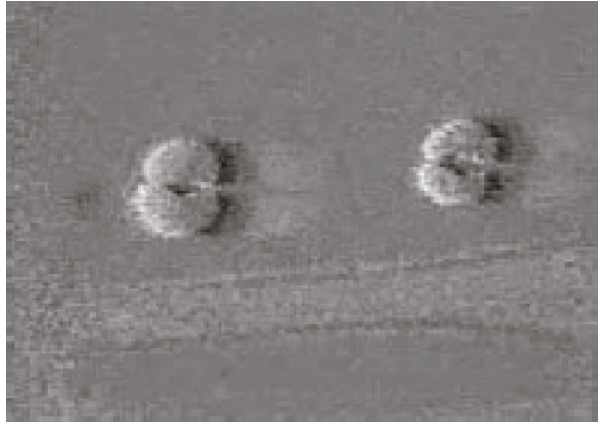

(b) 2nd set: useful component

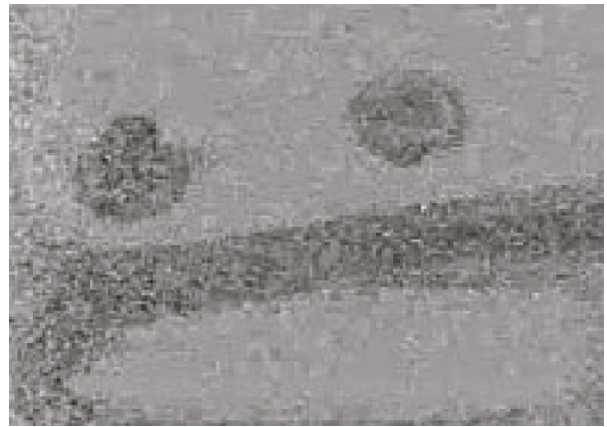

(d) 4th set: useful component

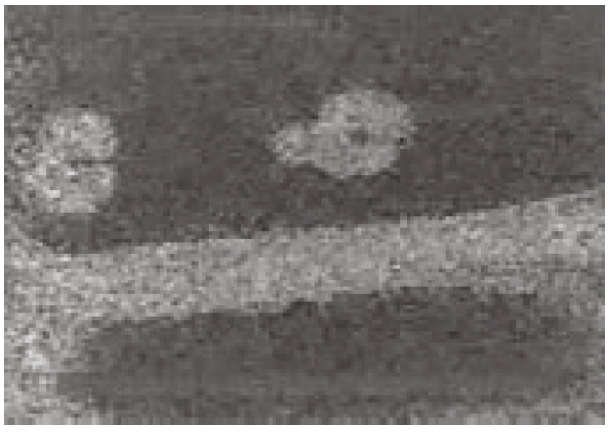

(f) 6th set: useful component

FIGURE 6: Independent components showing the informative signals, extracted from the in-study sets of magneto-optic images by the proposed approach.

BSS and ICA could be very helpful to practitioners to recover the unknown independent sources and it could be very useful in order to enhance the quality of MOI [16]. The aim is bringing out the rivets against the background, in order to ease the application of a further morphological operator able to isolate the rivet itself. In fact, we considered five sources of signals to be separated within the sets of images: source of mazes, source of bubbles, source of measure noise, source of environment random noise, and finally source of useful signal. In the general framework of ICA [9], a signal $\mathbf{x}(\cdot)$ in the time- or space domain is the result of mixing the records of $j$ different sources. Moreover, was $\mathbf{s}(\cdot)=$ $\left\{s_{1}(\cdot) ; s_{2}(\cdot) ; \ldots ; s_{j}(\cdot)\right\}$ with the set of the unknown $j$ source signals, $\mathbf{x}(\cdot)$ can be written as

$$
\mathbf{x}=\left[\begin{array}{cccc}
a_{11} & a_{12} & \ldots & a_{1 j} \\
a_{21} & a_{22} & \ldots & a_{2 j} \\
\ldots & \ldots & \ldots & \ldots \\
a_{j 1} & a_{j 2} & \ldots & a_{j j}
\end{array}\right] \mathbf{s}^{T}
$$

Under some general hypotheses, it is possible to recover the set of $j$ sources by calculating a suitable mixing matrix A, 


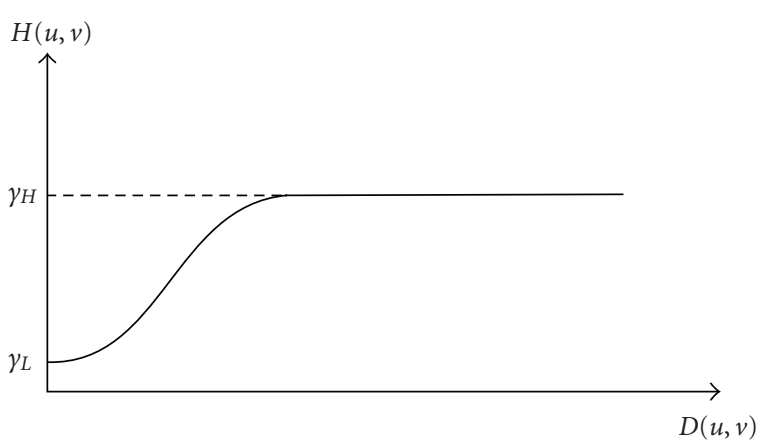

Figure 7: Cross-section of homomorphic filter function.

that is, the matrix with elements $a_{k h}$. Once the matrix $\mathbf{A}$ is calculated, it is possible to obtain its inverse $\mathbf{A}^{-1}$ and retrieve the independent components (ICs) having non-Gaussian distributions [17]. The fixed point algorithm [17] has been exploited in order to calculate and extract the independent components from each one of the in-study sets. Figure 6 shows results of our experimentations. Our analysis has been based on SNR evaluation since it relates the power of signal useful to the noise of the acquisition system. It can be considered as a measure of sensitivity performance and it has a remarkable importance in many applications in NDE. Within this framework, SNR is a key parameter for evaluation of proposed image processing and its importance cannot be underestimated. The SNR directly affects error probability and ultimately the detection of flaws in NDE. Within this framework, we propose the increment of SNR compared with the original SNR of the proposed sets in Table 2. The averaged SNR is more or less the same for the different sets, with a small decrement for the latter set. SNR is usually taken to indicate an average signal-to-noise ratio, as it is possible that (near) instantaneous signal-to-noise ratios would be considerably different. The concept can be understood as normalizing the noise level to $1(0 \mathrm{~dB})$ and measuring how far the signal "stands out." Finally let us note that, according to the method for calculating the SNR [15], it depends on the range of values given by the filtered images: thus, it is not important to consider the values of SNR as its increment due to preprocessing procedure.

The performances shown by the application of the ICA are generally remarkable in terms of increment of the SNR, but the evaluation of the reliability of the proposed method must be carried out by a joint consideration of the SNR increment itself with the assessment provided by a visual inspection of the useful independent components. In fact, the 5th set shows a result which can be assessed as an irregularity by a visual inspection, in spite of the SNR increment. The failure was expected, since the rivets depicted within the selected frames, as above explained, are different or in varying number. It introduces an irregularity in evaluating the mixing matrix and therefore its inverse demixing matrix, during the calculation procedure of the independent components. The result is a sort of mirroring the depicted rivets within the useful component. Really, it is the effect due to the superimposition of the different objects visualized within the different frames.

3.3. AHF for Enhancing MOI. The aim of image enhancement is to improve the interpretability or the perception of information in images for human viewers or to provide the "better" input for other automated image processing techniques. But, unfortunately, there is no general rule or any mathematical criterion for determining what is "good" image enhancement when it comes to the human perception. If the image looks good, it is good. This section considers the homomorphic filtering for the measurement of the degree of the enhancement of images in NDT\&E. Classical theory about filtering makes use of linear filters for the improvement of SNR. Our implementation regards a nonlinear system based on a generalized principle of linearity. White and black images can be represented by means of a 2 -variable system. Images are composed by reflection of the light from physical objects. The process of realization of an image can be modelled like a product about a lighting $\left(f_{i}\right)$ and a reflection $\left(f_{r}\right)$ function [18]:

$$
f(x, y)=f_{i}(x, y) \cdot f_{r}(x, y) .
$$

This equation cannot be used to operate separately and directly on the frequency components of illumination and reflectance, because the Fourier transform of the product of the functions is not separable. So, (3) can not be expressed as:

$$
\Im\{f(x, y)\}=\mathfrak{I}\left\{f_{i}(x, y)\right\} \cdot \Im\left\{f_{r}(x, y)\right\},
$$

where $\mathfrak{I}$ is the Fourier transform operator. Now, let us suppose that an image $f(x, y)$ can be defined in a way so that

$$
\begin{aligned}
& \mathfrak{I}\{z(x, y)\}=Z(u, v)=\mathfrak{I}\{\ln f(x, y)\} \\
& =\Im\left\{\ln \left(f_{i}(x, y)\right)\right\}+\Im\left\{\ln \left(f_{r}(x, y)\right)\right\} .
\end{aligned}
$$

For the sake of simplicity, please let us denote $F_{i}(u, v)=$

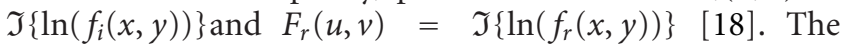
function $Z(u, v)$ can be processed by means of a filter function $H(u, v)$ and can be expressed as

$$
\begin{aligned}
S(u, v) & =H(u, v) \cdot Z(u, v) \\
& =H(u, v) \cdot F_{i}(u, v)+H(u, v) \cdot F_{r}(u, v),
\end{aligned}
$$

where $S(u, v)$ is the Fourier transform of the result. In the spatial domain $s(x, y)=\mathfrak{I}^{-1}\{S(u, v)\}=\mathfrak{I}^{-1}\{H(u, v)$. $\left.F_{i}(u, v)\right\}+\mathfrak{I}^{-1}\left\{H(u, v) \cdot F_{r}(u, v)\right\}$ by letting $f_{i}^{\prime}(x, y)=$ $\mathfrak{I}^{-1}\left\{H(u, v) \cdot F_{i}(u, v)\right\}$ and $f_{r}^{\prime}(x, y)=\mathfrak{I}^{-1}\left\{H(u, v) \cdot F_{r}(u, v)\right\}$. Finally the equation becomes

$$
\begin{gathered}
s(x, y)=f_{i}(x, y)+f_{r}(x, y) \\
g(x, y)=e^{s(x, y)}=e^{f_{i}^{\prime}(x, y)}+e^{f_{i}^{\prime}(x, y)}=f_{i_{0}}(x, y) \cdot f_{r_{0}}(x, y),
\end{gathered}
$$

where $f_{i_{0}}(x, y)=e^{f_{i}^{\prime}(x, y)}$ and $f_{r_{0}}(x, y)=e^{f_{r}^{\prime}(x, y)}$ are the illumination and the reflectance components of the output 


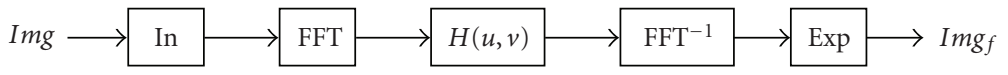

Figure 8: Block diagram of the AHF.

TABLE 2: Results after processing with ICA.

\begin{tabular}{lcccc}
\hline Inspected set & Visual comparison & Averaged SNR of original images $(\mathrm{dB})$ & SNR of useful IC $(\mathrm{dB})$ & SNR increment $(\mathrm{dB})$ \\
\hline 1 & Fair & 14.33 & 23.64 & 9.31 \\
2 & Good & 14.43 & 25.49 & 11.06 \\
3 & Optimal & 14.67 & 24.29 & 9.62 \\
4 & Sufficient & 14.31 & 21.47 & 7.34 \\
5 & Failure & 14.27 & 22.12 & 7.85 \\
6 & Sufficient & 13.73 & 18.88 & 5.15 \\
\hline
\end{tabular}

TABLE 3: Results of enhancing the sets of MO images.

\begin{tabular}{|c|c|c|c|c|}
\hline Inspected set & Averaged SNR of original images $(\mathrm{dB})$ & SNR MBF (dB) & SNR AHF (dB) & SNR ICA $(\mathrm{dB})$ \\
\hline 1 & 14.33 & 18.08 & 18.27 & 23.64 \\
\hline 2 & 14.43 & 16.31 & 17.40 & 25.49 \\
\hline 3 & 14.67 & 15.62 & 17.29 & 24.29 \\
\hline 4 & 14.31 & 19.50 & 17.91 & 21.47 \\
\hline 5 & 14.27 & 15.54 & 17.73 & 22.12 \\
\hline 6 & 13.73 & 18.07 & 18.27 & 18.88 \\
\hline
\end{tabular}

image. This method is based on a special class of systems known as homomorphic systems. The filter transfer function $H(u, v)$ is known as the homomorphic filter function:

$$
H(u, v)=\gamma_{L}+\frac{\gamma_{H}}{1+\left[D_{0} / \sqrt{u^{2}+v^{2}}\right]^{2 n}},
$$

where $\gamma_{L}$ and $\gamma_{H}$ are the lower and the higher frequency components, respectively, $D_{0}$ is the cut-off frequency, and $n$ defines the order of the filter. A good choice between the lower and the higher frequencies provides a dynamic range of the compression and enhancement [18]. $H(u, v)$ acts on the illumination and the reflectance components of the input image separately. The illumination component of an image is generally characterized by a slow spatial variations while the reflectance components vary abruptly, particularly at junctions of dissimilar objects. These characteristics actually lead to associate the low frequencies of the Fourier transform of the logarithm of an image with illumination and the high frequencies with reflectance [19].

This process of enhancement can be expressed by using the block diagram shown in Figure 8. Homomorphic filters use the discrete Fourier transforms (DFTs) as the core transform. Presently, in digital images, more efficient tools for transformations are used, such as fast Fourier transform (FFT) [19].

Homomorphic filter helps to have a good control over the illumination and the reflectance. In our algorithm, for a homomorphic filter of order 2, the cut-off frequency $D_{0}$ is adaptively calculated by inspecting the Fourier spectrum of the considered image and finding the maximum value of the spectrum. Then, we calculate the highest frequency showing a $3 \mathrm{~dB}$ loss as the final cut-off frequency. The input of our processor is the image img to be filtered. The order of the homomorphic filter is 2 . The output is the filtered image $\left(i m g_{f}\right)$.

A visual comparison of the proposed approach is shown in Figure 9. For each one of the collected image sets, the AHF has been applied to the central frame. The performance of the proposed AHF in terms of SNR is resumed in Table 3. Here, it is possible to make a quantitative comparison of the proposed approaches with MBF and ICA. Depending on the original image, the test value and the evaluation are not always correlated with the impression of quality of a subjective observation.

\section{Conclusions}

In this paper, the application of ICA for enhancing the quality of magneto-optic images has been discussed and compared with AHF and MBF. The MOI inspection technique is subjected to a special kind of measurement noise and also bubbles, mazes and other background static noise, due to the tape itself, that invariably influence the quality of the acquired images. These noise sources can be thought of as disturbing signals, with Gaussian and/or non-Gaussian probability density distributions, convolved with the useful signal. Accordingly, the practically relevant need of suitably denoising MO images can be approached as a problem of BSS. To deal with it, we decide to separate the underlying components of the signal by making use of a well-known algorithmic implementation of ICA. Comparing the performances of different algorithms, as reported in Table 3, we find that the standard performance of ICA is higher 


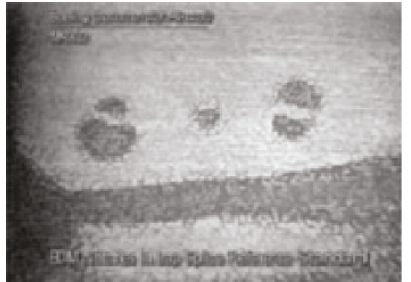

Frame 37: source

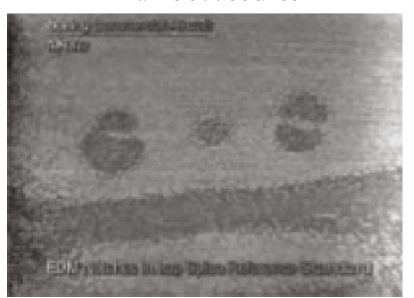

Frame 37: filtering by AHF

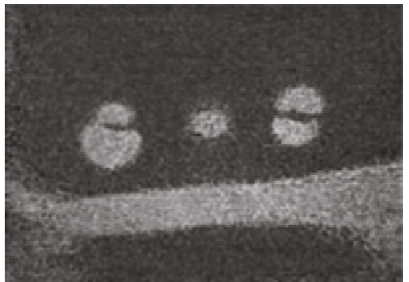

Frame 37: filtering by MBF

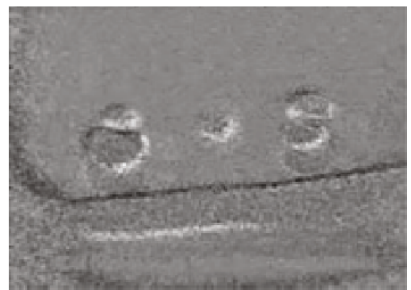

ICA result

(a) 1st set

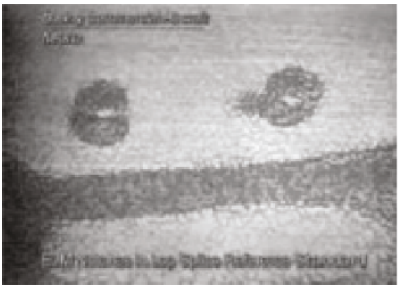

Frame 79: source

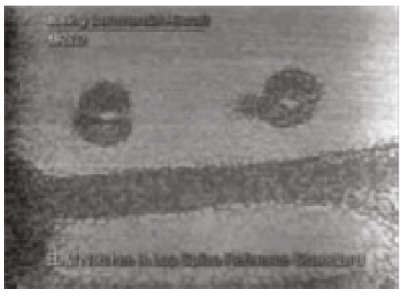

Frame 79: filtering by AHF

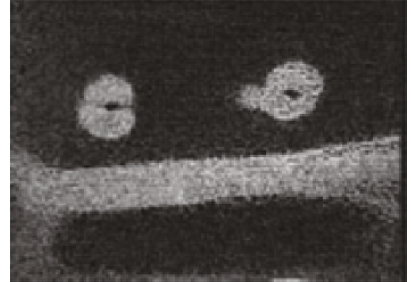

Frame 79: filtering by MBF

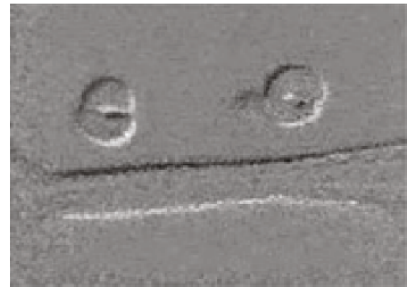

ICA result

(c) 3rd set

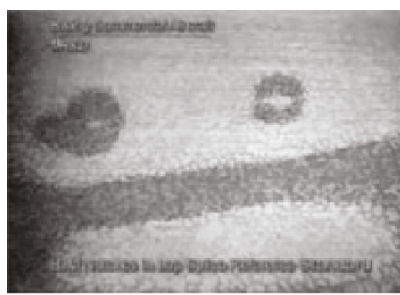

Frame 129: source

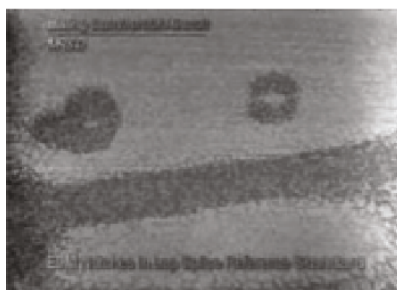

Frame 129: filtering by AHF

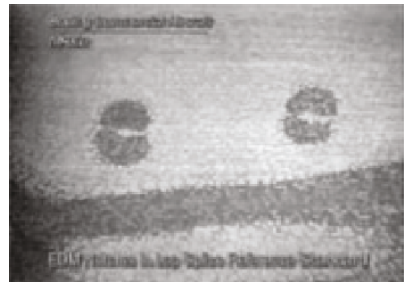

Frame 54: source

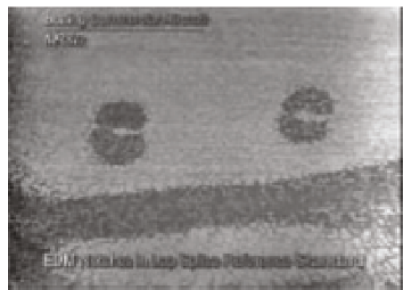

Frame 54: filtering by AHF

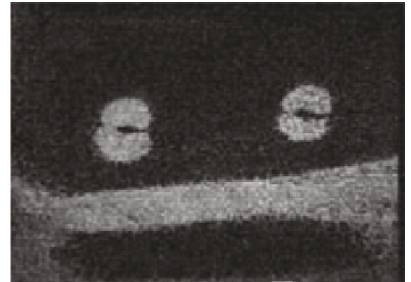

Frame 54: filtering by MBF

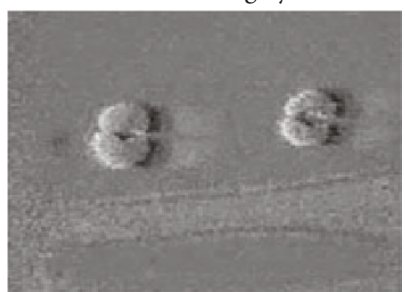

ICA result

(b) 2nd set

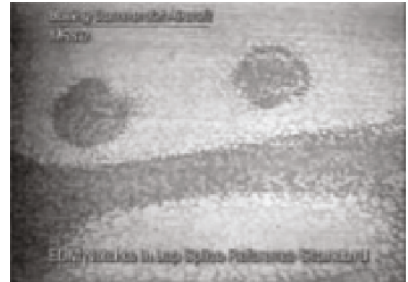

Frame 119: source

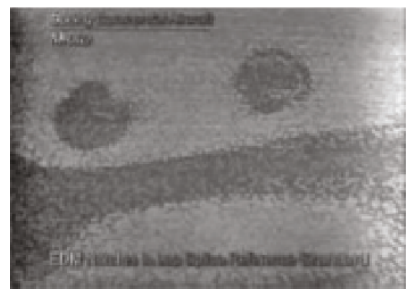

Frame 119: filtering by AHF

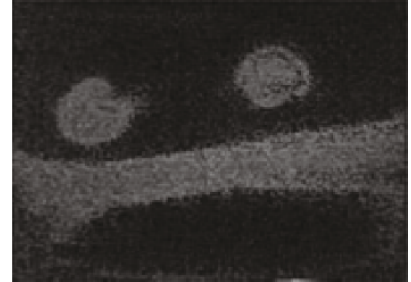

Frame 119: filtering by MBF

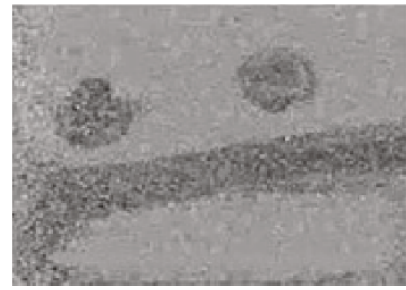

ICA result

(d) 4th set

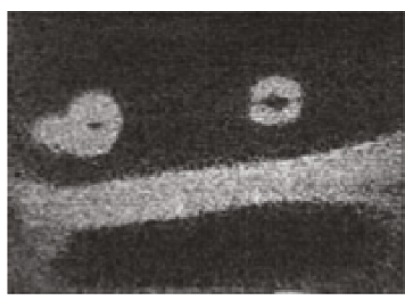

Frame 129: filtering by MBF

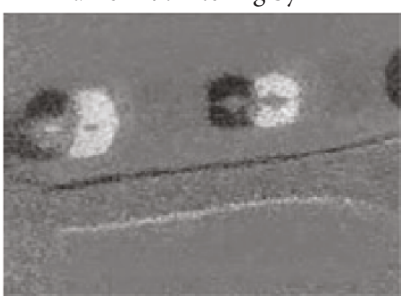

ICA result

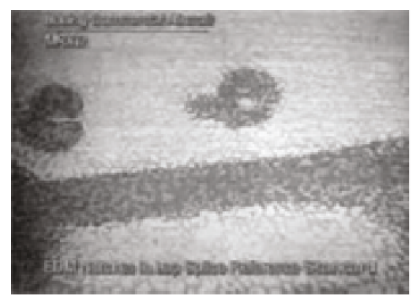

Frame 170: source

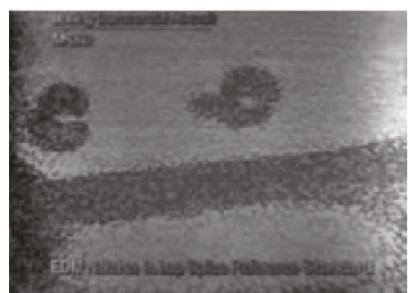

Frame 170: filtering by AHF

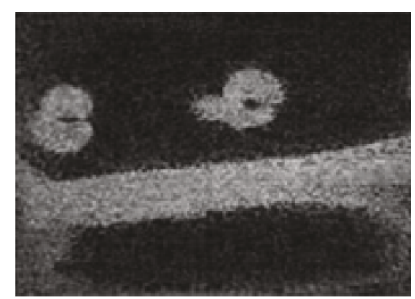

Frame 170: filtering by MBF

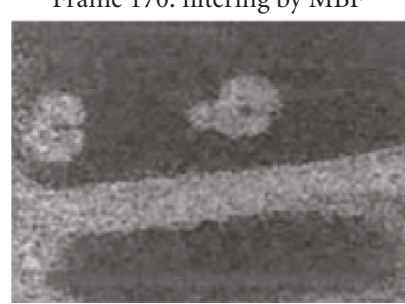

ICA result

(e) 5 th set

(f) 6th set

FIgure 9: Comparison of the performance for MBF, AHF, and ICA. 
or, at least, comparable with the MBF. ICA retrieves highly denoised images, in which rivets are well defined and highlighted. We claim that this is a noteworthy result, considering that the MBF is a filtering technique known to be particularly efficient in enhancing the quality of magneto-optic images. ICA averagely provided better results than AHF. Besides, the comparison with the other image processing methodologies showed ICA to be successful in increasing the SNR of the source images, so ICA filtering can help the human operator to detect defects more efficiently. Indeed, whereas MBF and AHF achieved an average improvement of the SNR of $4 \mathrm{dBs}$, ICA was able to enhance the quality of the images with averaged SNR increment of about $8 \mathrm{dBs}$ (refer to Table 3 for comparisons). Therefore, the ICA can be considered as a useful and reliable method for MOI preprocessing, in spite of the exploitation of ICA as an image filtering technique able to give a direct representation of the separate components. The weak point of ICA appears when the images included in the available set portray a different number of objects. Anyway, the presented results suggest the possibility of using ICA as a preprocessing method alternative to other images filtering procedures. Evaluation of the proposed algorithms using additional data (test for a large range of scanning velocity values) is under way. Future work suggests the possibility to test real-time processing by hardware implementation. Such a system can be directly connected to the MO imager and process data as they are acquired. Finally, the use of classification algorithms to identify regions with cracks is under investigation.

\section{Nomenclature}

\begin{tabular}{|c|c|}
\hline : & Angle of rotating light \\
\hline$\theta_{f}:$ & Incident angle of light \\
\hline $\mathrm{K}:$ & Wave vector of the incident light \\
\hline M: & $\begin{array}{l}\text { Local state of magnetization of the } \\
\text { sensor }\end{array}$ \\
\hline & Sensor thickness \\
\hline & Signal in time or spatial domain \\
\hline & $\begin{array}{l}\text { Transfer function of the Homomorphic } \\
\text { filter }\end{array}$ \\
\hline L: & $\begin{array}{l}\text { Lower frequency component of the } \\
\text { homomorphic filter }\end{array}$ \\
\hline$\gamma_{H}:$ & $\begin{array}{l}\text { Higher-frequency component of the } \\
\text { homomorphic filter }\end{array}$ \\
\hline$D_{0}:$ & $\begin{array}{l}\text { Cut-off frequency of the homomorphic } \\
\text { filter }\end{array}$ \\
\hline & Order of the homomorphic filter. \\
\hline
\end{tabular}

\section{References}

[1] Y. Yin, G. Y. Tian, G. F. Yin, and A. M. Luo, "Defect identification and classification for digital X-ray images," Applied Mechanics and Materials, vol. 10-12, pp. 543-547, 2008.

[2] S. Yamada, M. Katou, M. Iwahara, and F. P. Dawson, "Eddy current testing probe composed of planar coils," IEEE Transactions on Magnetics, vol. 31, no. 6, pp. 3185-3187, 1995.
[3] C. F. Morabito, "Independent component analysis and feature extraction techniques for NDT data," Materials Evaluation, vol. 58, no. 1, pp. 85-92, 2000.

[4] L. Udpa and S. S. Udpa, "Application of signal processing and pattern recognition techniques to inverse problems in NDE," International Journal of Applied Electromagnetics and Mechanics, vol. 8, no. 1, pp. 99-117, 1997.

[5] M. Cacciola, A. Gasparics, F. C. Morabito, M. Versaci, and V. Barrile, "Advances in signal processing to reduce lift-off noise in eddy current tests," PIERS Online, vol. 3, no. 4, pp. 517-521, 2007.

[6] S. Simms, "MOI: magneto-optic/eddy current imaging," Materials Evaluation, vol. 51, no. 5, pp. 529-532, 1993.

[7] G. L. Fitzpatrick, "Flaw Imaging in Ferrous and Nonferrous Materials Using Magneto-Optic Visualization," US Patents no. 4,755,752, 1988.

[8] G. L. Fitzpatrick, D. K. Thome, R. L. Skaugset, E. Y. C. Shih, and W. C. L. Shih, "Magneto-optic/eddy current imaging of aging aircraft: a new NDI technique," Materials Evaluation, vol. 51, no. 12, pp. 1402-1407, 1993.

[9] A. Hyvarinen, J. Karhunen, and E. Oja, Independent Component Analysis, John Wiley \& Sons, New York, NY, USA, 2001.

[10] G. Yang, G. Y. Tian, P. W. Que, and T. L. Chen, "Independent component analysis-based feature extraction technique for defect classification applied for pulsed eddy current NDE," Research in Nondestructive Evaluation, vol. 20, no. 4, pp. 230245, 2009.

[11] G. Y. Tian, A. Sophian, D. Taylor, and J. Rudlin, "Waveletbased PCA defect classification and quantification for pulsed eddy current NDT," IEE Proceedings: Science, Measurement and Technology, vol. 152, no. 4, pp. 141-148, 2005.

[12] P. Ramuhalli, F. Yuan, U. Park, J. Slade, and L. Udpa, "Enhancement of magneto-optic images," in Proceedings of the International Workshop on Electromagnetic Nondestructive Evaluation, p. 199, 2003.

[13] U. Park, L. Udpa, and G. C. Stockman, "Motion-based filtering of magneto-optic imagers," Image and Vision Computing, vol. 22, no. 3, pp. 243-249, 2004.

[14] K. Zvezdin and V. Kotov, "Modern magnetooptics and magnetooptical materials," Journal of the Optical Society of America B, vol. 22, no. 1, article 187, 1997.

[15] I. T. Young, J. J. Gerbrands, and L. J. van Vliet, Fundamentals of Image Processing, Delft University of Technology, Delft, The Netherlands, 1995.

[16] T.-W. Lee, M. Girolami, and T. J. Sejnowski, "Independent component analysis using an extended infomax algorithm for mixed subgaussian and supergaussian sources," Neural Computation, vol. 11, no. 2, pp. 417-441, 1999.

[17] A. Hyvärinen, "Fast and robust fixed-point algorithms for independent component analysis," IEEE Transactions on Neural Networks, vol. 10, no. 3, pp. 626-634, 1999.

[18] V. I. Ponomarev and A. B. Pogrebniak, "Image enhancement by homomorphic filters," in Applications of Digital Image Processing XVIII, vol. 2564 of Proceedings of SPIE, pp. 153-159, San Diego, Calif, USA, July 1995.

[19] R. C. Gonzales and R. E. Woods, Digital Image Processing, Addison Wesley, San Francisco, Calif, USA, 1993. 